\title{
A direct proof of Jauregui-Tsallis' conjecture
}

\author{
A. Plastino and M. C. Rocca \\ Departamento de Física-CCT-IFLP- CONICET \\ Fac. de Ciencias Exactas, Universidad Nacional de La Plata \\ C.C. 67 (1900) La Plata, Argentina \\ December 1, 2010
}

\begin{abstract}
We give here direct proof of a recent conjecture of Jauregui and Tsallis about a new representation of Dirac's delta distribution by means of q-exponentials. The proof is based in the use of tempered ultradistributions' theory.
\end{abstract}




\section{Introduction}

Empirical analysis suggests that power-law behavior in the (observed) distribution of some quantity is quite frequent in nature. Indeed, systems statistically described by power-law probability distributions (PLD) are rather ubiquitous [1] and thus of perennial interest [2, 3, 4]. Critical phenomena are just a conspicuous example [5]. Many objects that come in different sizes have a self-similar power-law distribution of their relative abundance over large size-ranges, from cities to words to meteorites [1]. More specifically, one often confronts a particularly important scenario: measuring real data distributed according to a q-Gaussian probability law, a special kind of powerlaw probability distribution function $(\mathrm{PDF})$, that is, a power-law distribution that maximizes the so-called Tsallis' information measure $H_{q}$ under variance constraint, with

$$
H_{q}(f)=\frac{1}{1-q}\left(1-\int_{-\infty}^{+\infty} f(x)^{q} d x\right)
$$

Intense activity revolves around this information measure, with hundreds of papers devoted to the study of its properties and development of applications in diverse scientific fields (see for instance, as a small sample, [3, 4, 6, 17, 8, 
9, 10, and references therein). The present effort is to be included in such context.

Tsallis and Jauregui have recently conjectured that, via probability distributions that maximize $H_{q}$ (called q-exponential functions) an interesting representation of the Dirac Delta distribution $\delta_{q}(x)$ can be given [11]. However, they could not prove their conjecture and used numerical experiments that suggest its validity. In the wake of this achievement, Chevreuil, Plastino and Vignat [12] provided a rigorous mathematical approach to the problem and proved the conjecture by recourse to the notion of superstatistics. Here we tackle again the subject and present a direct, structurally simpler proof by appealing to tempered ultradistributions' theory.

\section{The q-exponential function}

Statistical Mechanics' most notorious and renowned probability distribution is that deduced by Gibbs for the canonical ensemble [13, 14, usually referred to as the Boltzmann-Gibbs equilibrium distribution

$$
p_{G}(i)=\frac{\exp \left(-\beta E_{i}\right)}{Z_{B G}},
$$


with $E_{i}$ the energy of the microstate labeled by $i, \beta=1 / k_{B} T$ the inverse temperature, $k_{B}$ Boltzmann's constant, and $Z_{B G}$ the partition function. The exponential term $F_{B G}=\exp (-\beta E)$ is called the Boltzmann-Gibbs factor. Recently Beck and Cohen [7] have advanced a generalization, called "superstatistics", of this BG factor, assuming that the inverse temperature $\beta$ is a stochastic variable. The generalized statistical factor $F_{G S}$ is thus obtained as the multiplicative convolution

$$
F_{G S}=\int_{0}^{\infty} \frac{d \beta}{\beta} f(\beta) \exp (-\beta E),
$$

where $f(\beta)$ is the density probability of the inverse temperature. As stated above, $\beta$ is the inverse temperature, but the integration variable may also be any convenient intensive parameter. Superstatistics, meaning "superposition of statistics", takes into account fluctuations of such intensive parameters. Beck and Cohen also show that if $f(\beta)$ is a Gamma distribution, a special kind of thermostatistics arises, called nonextensive thermostatistics, a very active field, with applications to several scientific disciplines [8, 9, 10, 11]. In working in a nonextensive framework, one has to deal with power-law distributions called q-Gaussians, that maximize Tsallis' information measure subject to appropriate constraints, with $q \neq 1$ a real positive parameter called the nonextensivity index. More precisely, in the case of the celebrated 
canonical distribution, there is only one constraint, the energy $E$, i.e., $\langle E\rangle=$ $K$ ( $K$ a positive constant) and the equilibrium canonical distribution writes

$$
f_{q}(x)=\frac{1}{Z_{q}}\left(1-(1-q) \beta_{q} E\right)_{+}^{\frac{1}{1-q}},
$$

with $(x)_{+}=\max (0, x)$ and $\beta_{q}$ and $Z_{q}$ standing for the nonextensive counterparts of $\beta$ and $Z_{B G}$ above. Defining the $q$-exponential function as

$$
e_{q}(x)=(1+(1-q) x)_{+}^{\frac{1}{1-q}}
$$

allows to rewrite the equilibrium distribution in the more natural way

$$
f_{q}(x)=\frac{1}{Z_{q}} e_{q}(-\beta E) .
$$

It is a classical result that as $q \rightarrow 1$, Tsallis entropy reduces to Shannon's entropy

$$
H_{1}(f)=-\int_{-\infty}^{+\infty} f(x) \log f(x) .
$$

Accordingly, the $q$-exponential function converges to the usual exponential function. 


\section{Proof of Jauregui-Tsallis' conjecture}

\subsection{Preliminaries}

The problems of characterizing analytic functions whose boundary values are elements of the spaces of distributions, or, conversely, of finding representations of elements of the quoted spaces of generalized functions by analytic functions have a long history. Numerous papers have been written concerning ultradistribution spaces of Sebastiao e Silva [16. Such spaces are related to the solvability and the regularity problems of partial differential equations. Because of this relation, the study of the structural problems as well as problems of various operations and integral transformations in this setting is interesting in itself.

Thus, an analysis of spaces of distributions considered as boundary values of analytic functions having appropriate growth estimates, is of great value. One wishes to deal, in particular, with the Dirac's integral representation in ultradistribution spaces, with the convolution of tempered ultradistributions and ultradistributions of exponential type (in Quantum Field Theory), and with the integral transforms of tempered ultradistributions, of which the best known is the Fourier complex transformation. Recourse to such stuff 
will pave the way for our proof below. Some explanatory material that should help to understand this proof is given in Appendix I and Appendix II.

\subsection{Proof}

Our starting point is to consider $e_{q}(i k x)$ for $1<q<2$ and $k$ a real number:

$$
e_{q}(i k x)=[1+i(1-q) k x]^{\frac{1}{1-q}}
$$

Then $e_{q}(i k x)$ is the cut along the real k-axis of the tempered ultradistribution:

$$
E_{q}(i k x)=\{H(x) H[\Im(k)]-H(-x) H[-\Im(k)]\}[1+i(1-q) k x]^{\frac{1}{1-q}}
$$

where $H(x)$ is the Heaviside's step function. In (3.2) $k$ is a complex variable.

According to [15] we have the following formula:

$$
\int_{0}^{\infty} \frac{x^{\mu-1}}{(1+\beta x)^{\nu}} d x=\beta^{-\mu} \frac{\Gamma(\mu) \Gamma(\nu-\mu)}{\Gamma(\nu)}
$$

$0<\Re(\mu)<\Re(\nu),|\arg \beta|<\pi$, from which we deduce:

$$
\int_{0}^{\infty}(x+\beta)^{\nu} d x=-\frac{\beta^{1+\nu}}{1+\nu}
$$

$\Re(\nu)<-1,|\arg \beta|<\pi$ Let $F_{q}(k)$ be given by:

$$
F_{q}(k)=\int_{-\infty}^{\infty} E_{q}(i k x) d x
$$


Then:

$$
\begin{gathered}
F_{q}(k)=H[\Im(k)][(1-q) i k]^{\frac{1}{1-q}} \int_{0}^{\infty}\left[x+\frac{1}{(1-q) i k}\right]^{\frac{1}{1-q}} d x- \\
H[-\Im(k)][(q-1) i k]^{\frac{1}{1-q}} \int_{0}^{\infty}\left[x+\frac{1}{(q-1) i k}\right]^{\frac{1}{1-q}} d x
\end{gathered}
$$

Using (3.4) we obtain for (3.6) $(1<q<2)$

$$
\begin{gathered}
F_{q}(k)=-H[\Im(k)] \frac{1}{(2-q) i k}-H[-\Im(k)] \frac{1}{(2-q) i k}= \\
\frac{1}{2-q}\left(-\frac{1}{i k}\right)=\frac{2 \pi}{2-q} \delta(k)
\end{gathered}
$$

We reach an important milestone here. Formula (3.7) is the proof of JaureguiTsallis' conjecture for $k$ complex..

The idea is now to consider the real axis in the variable $k$. To this effect we note that

$$
\begin{gathered}
\frac{2 \pi}{2-q} \phi(0)=\oint_{\Gamma} F_{q}(k) \phi(k) d k= \\
\int_{-\infty}^{\infty} \lim _{\epsilon \rightarrow 0^{+}}\left\{\int_{0}^{\infty}[1+(1-q) i(k+i \epsilon) x]^{\frac{1}{1-q}} d x+\right. \\
\left.\int_{-\infty}^{0}[1+(1-q) i(k-i \epsilon) x]^{\frac{1}{1-q}} d x\right\} \phi(k) d k
\end{gathered}
$$


where $\phi(k)$ is an analytic test function rapidly decreasing (See Appendix II)

. From (3.8) we obtain:

$$
\begin{gathered}
\int_{-\infty}^{\infty} e_{q}(i k x) d x=\lim _{\epsilon \rightarrow 0^{+}}\left\{\int_{0}^{\infty}[1+(1-q) i(k+i \epsilon) x]^{\frac{1}{1-q}} d x+\right. \\
\left.\int_{-\infty}^{0}[1+(1-q) i(k-i \epsilon) x]^{\frac{1}{1-q}} d x\right\}=\frac{2 \pi}{2-q} \delta(k)
\end{gathered}
$$

We have reached our goal. Formula (3.9) is the proof of Jauregui-Tsallis' conjecture on the real axis.

\section{Conclusions}

In this paper we have straightforwardly proved the Jauregui-Tsallis' conjecture by recourse to tempered ultradistributions' theory, on the complex plane and on the real axis. We remark on the fact that the vital ingredient here are the so-called q-exponential distributions, that play a significant role in statistical mechanics as maximizers of Tsallis' entropy under variance constraint. Thus we are in a rather curious position of having obtained the proof of a mathematical conjecture inspired by thermodynamic's ideas. 


\section{Appendix I: Distributions of Exponential}

\section{Type}

For the reader's benefit we briefly review the main properties of Tempered Ultradistributions.

Notations. The notations are almost textually taken from Ref. [17]. Let $\mathbb{R}^{n}$ (res. $\mathbb{C}^{\boldsymbol{n}}$ ) be the real (resp. complex) n-dimensional space whose points are denoted by $x=\left(x_{1}, x_{2}, \ldots, x_{n}\right)$ (resp $\left.z=\left(z_{1}, z_{2}, \ldots, z_{n}\right)\right)$. We shall use the notations:

(i) $x+y=\left(x_{1}+y_{1}, x_{2}+y_{2}, \ldots, x_{n}+y_{n}\right) ; \alpha x=\left(\alpha x_{1}, \alpha x_{2}, \ldots, \alpha x_{n}\right)$

(ii) $x \geqq 0$ means $x_{1} \geqq 0, x_{2} \geqq 0, \ldots, x_{n} \geqq 0$

(iii) $x \cdot y=\sum_{j=1}^{n} x_{j} y_{j}$

$(\mathrm{iV})|x|=\sum_{j=1}^{n}\left|x_{j}\right|$

Let $\mathbb{N}^{n}$ be the set of $n$-tuples of natural numbers. If $p \in \mathbb{N}^{n}$, then $p=$ $\left(p_{1}, p_{2}, \ldots, p_{n}\right)$, and $p_{j}$ is a natural number, $1 \leqq j \leqq n . p+q$ denote $\left(p_{1}+\right.$ $\left.q_{1}, p_{2}+q_{2}, \ldots, p_{n}+q_{n}\right)$ and $p \geqq q$ means $p_{1} \geqq q_{1}, p_{2} \geqq q_{2}, \ldots, p_{n} \geqq q_{n} . x^{p}$ means $x_{1}^{p_{1}} x_{2}^{p_{2}} \ldots x_{n}^{p_{n}}$. We shall denote by $|p|=\sum_{j=1}^{n} p_{j}$ and by $D^{p}$ we denote the differential operator $\partial^{p_{1}+p_{2}+\ldots+p_{n}} / \partial x_{1}{ }^{p_{1}} \partial x_{2}{ }^{p_{2}} \ldots \partial x_{n}{ }^{p_{n}}$

For any natural $k$ we define $x^{k}=x_{1}^{k} x_{2}^{k} \ldots x_{n}^{k}$ and $\partial^{k} / \partial x^{k}=\partial^{n k} / \partial x_{1}^{k} \partial x_{2}^{k} \ldots \partial x_{n}^{k}$ 
The space $\mathcal{H}$ of test functions such that $e^{p|x|}\left|D^{q} \phi(x)\right|$ is bounded for any $\mathrm{p}$ and $\mathrm{q}$ is defined ( ref.[17] ) by means of the countably set of norms:

$$
\|\hat{\phi}\|_{p}=\sup _{0 \leq q \leq p, x} e^{p|x|}\left|D^{q} \hat{\phi}(x)\right| \quad, \quad p=0,1,2, \ldots
$$

According to reference[19] $\mathcal{H}$ is a $\mathcal{K}\left\{\boldsymbol{M}_{\boldsymbol{p}}\right\}$ space with:

$$
M_{p}(x)=e^{(p-1)|x|} \quad, \quad p=1,2, \ldots
$$

$\mathcal{K}\left\{e^{(p-1)|x|}\right\}$ satisfies condition $(\mathcal{N})$ of Guelfand ( ref.[18] ). It is a countable Hilbert and nuclear space:

$$
\mathcal{K}\left\{e^{(p-1)|x|}\right\}=\mathcal{H}=\bigcap_{p=1}^{\infty} \mathcal{H}_{p}
$$

where $\mathcal{H}_{\boldsymbol{p}}$ is obtained by completing $\mathcal{H}$ with the norm induced by the scalar product:

$$
<\hat{\phi}, \hat{\psi}>_{p}=\int_{-\infty}^{\infty} e^{2(p-1)|x|} \sum_{q=0}^{p} D^{q} \overline{\hat{\phi}}(x) D^{q} \hat{\psi}(x) d x \quad ; \quad p=1,2, \ldots
$$

where $d x=d x_{1} d x_{2} \ldots d x_{n}$

If we take the usual scalar product:

$$
<\hat{\phi}, \hat{\psi}>=\int_{-\infty}^{\infty} \overline{\hat{\phi}}(x) \hat{\psi}(x) d x
$$

then $\mathcal{H}$, completed with $(\underline{5.5})$, is the Hilbert space $\boldsymbol{H}$ of square integrable functions. 
The space of continuous linear functionals defined on $\mathcal{H}$ is the space $\boldsymbol{\Lambda}_{\infty}$ of the distributions of the exponential type ( ref.[17] ).

The "nested space"

$$
H=\left(\mathcal{H}, \boldsymbol{H}, \Lambda_{\infty}\right)
$$

is a Guelfand's triplet ( or a Rigged Hilbert space [18] ).

In addition we have: $\mathcal{H} \subset \mathcal{S} \subset \boldsymbol{H} \subset \mathcal{S}^{\prime} \subset \boldsymbol{\Lambda}_{\infty}$, where $\mathcal{S}$ is the Schwartz space of rapidly decreasing test functions (ref[20]).

Any Guelfand's triplet $G=\left(\Phi, \boldsymbol{H}, \Phi^{\prime}\right)$ has the fundamental property that a linear and symmetric operator on $\mathbf{\Phi}$, admitting an extension to a self-adjoint operator in $\boldsymbol{H}$, has a complete set of generalized eigen-functions in $\boldsymbol{\Phi}^{\prime}$ with real eigenvalues.

\section{Appendix II: Tempered Ultradistributions}

The Fourier transform of a function $\hat{\phi} \in \mathcal{H}$ is

$$
\phi(z)=\frac{1}{2 \pi} \int_{-\infty}^{\infty} \bar{\phi}(x) e^{i z \cdot x} d x
$$

$\phi(z)$ is entire analytic and rapidly decreasing on straight lines parallel to the real axis. We shall call $\mathfrak{H}$ the set of all such functions.

$$
\mathfrak{H}=\mathcal{F}\{\mathcal{H}\}
$$


It is a $\mathcal{Z}\left\{\boldsymbol{M}_{\boldsymbol{p}}\right\}$ space ( ref.[19] ), countably normed and complete, with:

$$
M_{p}(z)=(1+|z|)^{p}
$$

$\mathfrak{H}$ is also a nuclear space with norms:

$$
\|\phi\|_{p n}=\sup _{z \in V_{n}}(1+|z|)^{p}|\phi(z)|
$$

where $V_{k}=\left\{z=\left(z_{1}, z_{2}, \ldots, z_{n}\right) \in \mathbb{C}^{n}:\left|I m z_{j}\right| \leqq k, 1 \leqq j \leqq n\right\}$

We can define the usual scalar product:

$$
<\phi(z), \psi(z)>=\int_{-\infty}^{\infty} \phi(z) \psi_{1}(z) d z=\int_{-\infty}^{\infty} \overline{\hat{\phi}}(x) \hat{\psi}(x) d x
$$

where:

$$
\psi_{1}(z)=\int_{-\infty}^{\infty} \hat{\psi}(x) e^{-i z \cdot x} d x
$$

and $d z=d z_{1} d z_{2} \ldots d z_{n}$

By completing $\mathfrak{H}$ with the norm induced by (6.5) we get the Hilbert space of square integrable functions.

The dual of $\mathfrak{H}$ is the space $\mathcal{U}$ of tempered ultradistributions ( ref.[17] ). In other words, a tempered ultradistribution is a continuous linear functional defined on the space $\mathfrak{H}$ of entire functions rapidly decreasing on straight lines parallel to the real axis.

The set $U=(\mathfrak{H}, \boldsymbol{H}, \mathcal{U})$ is also a Guelfand's triplet. 
Moreover, we have: $\mathfrak{H} \subset \mathcal{S} \subset \boldsymbol{H} \subset \mathcal{S}^{\prime} \subset \mathcal{U}$.

$\mathcal{U}$ can also be characterized in the following way ( ref.[17] ): let $\mathcal{A}_{\boldsymbol{\omega}}$ be the space of all functions $F(z)$ such that:

$\boldsymbol{I}$ - $F(z)$ is analytic for $\left\{z \in \mathbb{C}^{n}:\left|\operatorname{Im}\left(z_{1}\right)\right|>p,\left|\operatorname{Im}\left(z_{2}\right)\right|>p, \ldots,\left|\operatorname{Im}\left(z_{n}\right)\right|>\right.$ $p\}$.

$\boldsymbol{I} \boldsymbol{I}-F(z) / z^{p}$ is bounded continuous in $\left\{z \in \mathbb{C}^{n}:\left|\operatorname{Im}\left(z_{1}\right)\right| \geqq p,\left|\operatorname{Im}\left(z_{2}\right)\right| \geqq\right.$ $\left.p, \ldots,\left|\operatorname{Im}\left(z_{n}\right)\right| \geqq p\right\}$, where $p=0,1,2, \ldots$ depends on $F(z)$.

Let $\Pi$ be the set of all $z$-dependent pseudo-polynomials, $z \in \mathbb{C}^{n}$. Then $\mathcal{U}$ is the quotient space:

\section{$\boldsymbol{I I I}-\mathcal{U}=\mathcal{A}_{\omega} / \Pi$}

By a pseudo-polynomial we understand a function of $z$ of the form $\sum_{s} z_{j}^{s} G\left(z_{1}, \ldots, z_{j-1}, z_{j+1}, \ldots, z_{n}\right)$ with $G\left(z_{1}, \ldots, z_{j-1}, z_{j+1}, \ldots, z_{n}\right) \in \mathcal{A}_{\boldsymbol{\omega}}$

Due to these properties it is possible to represent any ultradistribution as ( ref.[17] ):

$$
F(\phi)=<F(z), \phi(z)>=\oint_{\Gamma} F(z) \phi(z) d z
$$

$\Gamma=\Gamma_{1} \cup \Gamma_{2} \cup \ldots \Gamma_{n}$ where the path $\Gamma_{j}$ runs parallel to the real axis from $-\infty$ to $\infty$ for $\operatorname{Im}\left(z_{j}\right)>\zeta, \zeta>p$ and back from $\infty$ to $-\infty$ for $\operatorname{Im}\left(z_{j}\right)<-\zeta$, $-\zeta<-p .(\Gamma$ surrounds all the singularities of $F(z))$.

Formula (6.6) will be our fundamental representation for a tempered ultra- 
distribution. Sometimes use will be made of "Dirac formula" for ultradistributions (Ref. [16]):

$$
F(z)=\frac{1}{(2 \pi i)^{n}} \int_{-\infty}^{\infty} \frac{f(t)}{\left(t_{1}-z_{1}\right)\left(t_{2}-z_{2}\right) \ldots\left(t_{n}-z_{n}\right)} d t
$$

where the "density" $f(t)$ is such that

$$
\oint_{\Gamma} F(z) \phi(z) d z=\int_{-\infty}^{\infty} f(t) \phi(t) d t
$$

While $F(z)$ is analytic on $\Gamma$, the density $f(t)$ is in general singular, so that the r.h.s. of (6.8) should be interpreted in the sense of distribution theory. Another important property of the analytic representation is the fact that on $\Gamma, F(z)$ is bounded by a power of $z$ (Ref. [17]):

$$
|F(z)| \leq C|z|^{p}
$$

where $C$ and $p$ depend on $F$.

The representation (6.6) implies that the addition of a pseudo-polynomial $P(z)$ to $F(z)$ do not alter the ultradistribution:

$$
\oint_{\Gamma}\{F(z)+P(z)\} \phi(z) d z=\oint_{\Gamma} F(z) \phi(z) d z+\oint_{\Gamma} P(z) \phi(z) d z
$$

But:

$$
\oint_{\Gamma} P(z) \phi(z) d z=0
$$


as $P(z) \phi(z)$ is entire analytic in some of the variables $z_{j}$ (and rapidly decreasing),

$$
\therefore \oint_{\Gamma}\{F(z)+P(z)\} \phi(z) d z=\oint_{\Gamma} F(z) \phi(z) d z .
$$




\section{References}

[1] M. Scroeder, Fractals, Chaos, Power Laws, (Freeman, NY, 1991).

[2] M. Gell-Mann and C. Tsallis, Eds. Nonextensive Entropy: Interdisciplinary applications (Oxford University Press, Oxford, 2004); C. Tsallis, Braz. J. of Phys 29 (1999) 1; J. Stat. Phys. 52 (1988) 479; A. Plastino and A. R. Plastino, Braz. J. of Phys., 29, (1999) 50.

[3] C. Vignat, A. Plastino, Phys. Lett. A 343, (2005) 411; 365 (2007) 370.

[4] C. Vignat and A. Plastino, Phys. Lett. A 360 (2006) 415.

[5] N. Goldenfeld, Lectures on phase transitions and the renormalization group (Addison-Wesley, NY, 1992).

[6] C. Tsallis, Introduction to nonextensive statistical mechanics (Springer, NY, 2009); See also http://tsallis.cat.cbpf.br/biblio.htm.

[7] C. Beck and E. G. D. Cohen, Physica A 322 (2003) 267.

[8] Gell-Mann M and Tsallis C, Eds. 2004 Nonextensive Entropy: Interdisciplinary applications (Oxford: Oxford University Press) and references therein; Plastino A R and Plastino A 1994 Phys. Lett. A 193140. 
[9] Kaniadakis G, Lissia M, and Rapisarda A., Eds., 2002 Nonextensive statistical mechanics and physical applications, Physica A (Special) 305, and references therein.

[10] A.R. Plastino, A. Plastino, Phys. Lett. A 193 (1994) 251.

[11] M. Jauregui, C. Tsallis, J. Math. Phys. 51, 063304 (2010),

[12] A. Chevreuil, A. Plastino, and C. Vignat J. Math. Phys. 51, 093502 $(2010)$

[13] Reif F 1965 Statistical and thermal physics (NY: McGraw-Hill); Pathria R K 1993 Statistical Mechanics (Exeter: Pergamon Press).

[14] Gibbs J W 1948 Elementary principles in statistical mechanics in Collected Works (New Haven: Yale University Press; R. B. Lindsay R B and Margenau H 1957 Foundations of physics (NY: Dover).

[15] L. S. Gradshtein and I. M. Ryzhik : Table of Integrals, Series, and Products. Sixth edition, Academic Press (2000).

[16] J. Sebastiao e Silva : Math. Ann. 136, 38 (1958).

[17] M. Hasumi: Tôhoku Math. J. 13, 94 (1961). 
[18] I. M. Gel'fand and N. Ya. Vilenkin : Generalized Functions Vol. 4. Academic Press (1964).

[19] I. M. Gel'fand and G. E. Shilov : Generalized Functions Vol. 2. Academic Press (1968).

[20] L. Schwartz: Théorie des distributions. Hermann, Paris (1966). 